\title{
Small Magnetic Toroid Antenna Imbedded in a Highly Conducting Half Space
}

\author{
George R. Swain \\ University of New Mexico, Albuquerque, N.Mex.
}

(Received August 3, 1964; revised November 16, 1964)

\begin{abstract}
The antenna considered consists of a small toroidal core of magnetic material, upon which a uniformly distributed driving winding is placed. Thin layers of insulation separate the winding from the core and from the highly conducting medium in which the antenna is situated. The external fields for the driven antenna are estimated using a static field solution employing toroidal coordinates. The driving-point admittance is then determined from these fields. The effective electric current moment of the antenna is estimated by duality with the electric current loop. The driving-point admittance and the effective moment are then combined to estimate the effective length and the relative effective area of a toroid antenna oriented such that the electric moment produced is parallel to the surface of the conducting half space. While the effective length of a tuned toroid antenna can be made much greater than the effective length of an electric-dipole-in-radome antenna, the effective areas of the two antenna types are of the same order of magnitude if the maximum exterior dimensions are equal.
\end{abstract}

\section{Introduction}

The need to communicate between subsurface points of the earth or between submerged submarines has provided an incentive for investigating antenna structures imbedded in a conducting medium. The antenna to be considered in this paper consists of a toroidal core of highly permeable magnetic material, upon which a uniformly distributed driving winding is placed. The winding is assumed to be insulated from the core and from the surrounding medium by thin layers of insulation. The toroid antenna is assumed to lie below the surface of a highly conducting half space, while the remaining half space is assumed to be a lossless dielectric with the same magnetic permeability. This configuration represents the earth or sea and the air adequately for some VLF or ELF applications. The axis of rotational symmetry of the toroid, which is also the direction of the electric current moment produced, is oriented parallel to the surface of the conducting medium, as shown in figure 1 . Moore and Blair [1961] and Wait [1961] have shown that this orientation of the electric moment produces transmission from the conducting medium into the dielectric half space. It is further assumed that the radius of the toroid is small compared to a skin depth in the conducting medium.

The driving-point admittance of the antenna will first be investigated using a static field solution of the wave equation in toroidal coordinates. Secondly, an estimate of the effective electric current moment produced by the antenna will be made. Finally, these results will be combined to estimate the effective length and the effective area of the antenna, enabling one to compare the performance of this antenna with other subsurface or surface antennas.

\section{Impedance Properties of the Toroid Antenna}

The driving-point admittance of the magnetic toroid antenna depends upon the fields created in its vicinity. Since the toroid considered is small compared to the skin depth in the conducting medium, a static solution for the fields will suffice. Also the nearness of the toroid to the surface of the conducting medium is ignored. This is permissible, since the conducting medium shields the antenna from distant points. The electric and magnetic fields within the toroidal core will be designated by $\overrightarrow{E_{c}}$ and $\overrightarrow{H_{c}}$; those external to the toroid by $\vec{E}$ and $\vec{H}$.

Before determining the admittance, one may outline the procedure to be used. The magnetic field $H_{c}$ inside the toroid links both the current $N I$ in the $N$ turns of the driving winding and the current $I_{m}$ induced in the medium and flowing through the toroid opening; the field is thus related to both currents. One observes that the voltage per turn $V \mid N$ may be determined in terms of the sum of the winding currents and the induced current, $N I+I_{m}$, by integrating the internal magnetic field $\mu_{c} H_{c}$ over the cross-sectional area of the core, and taking the time derivative of this flux. The induced current $I_{m}$ is not initially known, and it is desired to relate this quantity to a known one. One notes that the magnetic field $\vec{H}$ on the exterior surface of the toroid may be determined in terms of the induced current $I_{m}$. Using the static wave equation, one may then determine the field $\vec{H}$ everywhere outside the toroid, subject to this boundary condition. Then the electric field $\vec{E}$ may be determined in terms of the magnetic field $\vec{H}$, and finally, the voltage per turn $V / N$ may be obtained by integrating the electric field around a path linking the toroid. The result 
of these operations is to relate $I_{m}$ to $V / N$. Having a relation of $N I+I_{m}$ to $V / N$ and another relation of $I_{m}$ to $V / N$, one may combine the two to eliminate $I_{m}$. The result relates $I$ to $V$, and hence gives the drivingpoint admittance.

The procedure begins with the determination of the internal magnetic field $\vec{H}_{c}$ in terms of the driving current $N I$ and the induced current $I_{m}$. One first notes that the problem is symmetric in the azimuthal coordinate $\varphi$. The only significant component of the magnetic intensity $\vec{H}_{c}$ present is $H_{c \varphi}$, as all driving currents lie in planes nearly perpendicular to the $\varphi$ direction. Let $\rho$ be the distance from the axis of rotational symmetry of the toroid to a point inside the core. Then if one integrates $H_{c \varphi}$ along a flux line, only the currents $N I$ and $I_{m}$ are enclosed (neglecting displacement currents in the core, which is valid for the small toroid), one deduces that

$$
H_{c \varphi}=\frac{N I+I_{m}}{2 \pi \rho} .
$$

The voltage per turn $V \mid N$ may then be expressed (for $e^{j \omega t}$ time variations) as

$$
V \mid N=+j \omega \iint_{A_{c}} \mu_{c}\left(\frac{N I+I_{m}}{2 \pi \rho}\right) d a
$$

in which $\mu_{c}$ is the permeability of the core and $A_{c}$ is the area of the core cross section. Alternatively, one may write

$$
V / N=\frac{+j \omega \mu_{c} A_{c}}{2 \pi \bar{\rho}}\left(N I+I_{m}\right)
$$

where $\bar{\rho}$ is the effective magnetic radius of the toroid. For a toroid of circular cross section with radius $r_{0}$ whose center is at a radius $\rho_{0}$ from the axis of rotational symmetry, as shown in figure 1 , one finds

$$
\bar{\rho}=\frac{1}{2}\left(\rho_{0}+\sqrt{\rho_{0}^{2}-r_{0}^{2}}\right) ; \quad A_{c}=\pi r_{0}^{2} .
$$

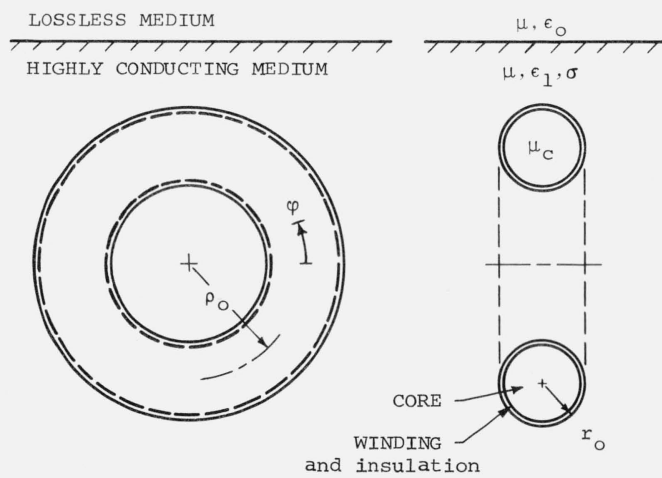

FIGURE 1. Orientation and dimensions of the toroid antenna.

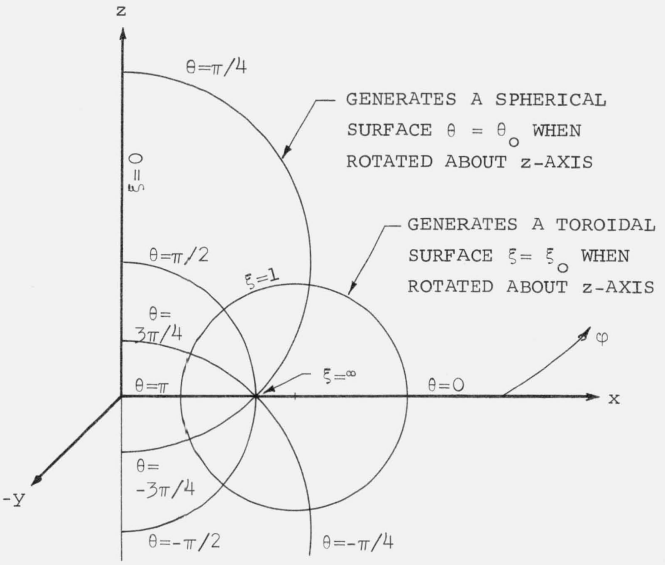

Figure 2. Toroidal coordinate system.

Equation (3) relates $V / N$ to the currents $N I$ and $I_{m}$. To find a relation between $V / N$ and $I_{m}$ alone, one begins by determining the magnetic field $\vec{H}$ outside the toroid. For the static case, this field must satisfy

$$
\vec{H}=H_{\varphi} \overrightarrow{a_{\varphi}}, \quad \nabla \times \nabla \times \vec{H}=0,
$$

subject to the boundary condition

$$
H_{\varphi}=\frac{I_{m}}{2 \pi \rho}
$$

for points on the outside surface of the toroid.

Toroidal coordinates $(\xi, \theta, \varphi)$ (see fig. 2) provide a convenient coordinate space, since the boundary surface may be specified as $\xi=\xi_{0}$, a surface of constant $\xi$. Toroidal coordinates are related to cylindrical coordinates according to

$$
\begin{aligned}
& \rho=\frac{a \sinh \xi}{\cosh \xi-\cos \theta} \\
& z=\frac{a \sin \theta}{\cosh \xi-\cos \theta} \\
& \varphi=\varphi,
\end{aligned}
$$

in which $a$ is a constant having the dimensions of length. The constants $\xi_{0}$ and $a$ are related to the dimensions $\rho_{0}$ and $r_{0}$ by:

$$
\begin{gathered}
\cosh \xi_{0}=\frac{\rho_{0}}{r_{0}} \\
a \operatorname{csch} \xi_{0}=r_{0} .
\end{gathered}
$$

Letting

$$
H_{\varphi}=(\cosh \xi-\cos \theta)^{1 / 2} U_{1}(\xi) U_{2}(\theta)
$$


in (5), one finds that

$$
\begin{gathered}
U_{1}^{\prime \prime}+\operatorname{coth} \xi U_{1}^{\prime}+\left(\frac{1}{4}-n^{2}-\operatorname{csch}^{2} \xi\right) U_{1}=0 \\
U_{2}^{\prime \prime}+n^{2} U_{2}=0 .
\end{gathered}
$$

The boundary condition (6), when expressed in toroidal coordinates, is

$$
H_{\varphi}\left(\xi_{0}, \theta\right)=\frac{I_{m}}{2 \pi a} \frac{\cosh \xi_{0}-\cos \theta}{\sinh \xi_{0}} .
$$

Equation (9) leads to sinusoidal functions. If these are to be continuous through the toroid opening at $\theta= \pm \pi, n$ must be an integer; further, if the functions are to be even in $\theta$ as the boundary condition (10) is, the functions are cosines. Equation (8) leads to associated Legendre functions of the first and second kind, of first order, and of half integer degree:

$$
P_{n-1 / 2}^{1}(\cosh \xi), \quad Q_{n-1 / 2}^{1}(\cosh \xi)
$$

(The definitions of the Legendre functions used in this paper are those of Erdelyi et al., [1953].) Only the functions of the first kind are suitable for representing a field outside a toroid, since the functions of the second kind increase without limit as $\xi$ goes to zero.

One may then express the external magnetic field in the form

$$
H_{\varphi}=\frac{I_{m}}{2 \pi a} \frac{(s-\cos \theta)^{1 / 2}}{\left(s_{0}^{2}-1\right)^{1 / 2}} \sum_{n=0}^{\infty} A_{n} \frac{P_{n-1 / 2}^{1}(s)}{P_{n-1 / 2}^{1}\left(s_{0}\right)} \cos n \theta
$$

where

$$
\begin{aligned}
& s=\cosh \xi, \\
& s_{0}=\cosh \xi_{0},
\end{aligned}
$$

and the $A_{n}$ are coefficients to be determined from the boundary condition. The constants in $s_{0}$ are included to simplify the statement of the boundary condition, which by (10) and (11) is

$$
\left(s_{0}-\cos \theta\right)^{1 / 2}=\sum_{n=0}^{\infty} A_{n} \cos n \theta .
$$

The evaluation of the constants $A_{n}$ is thus a Fourier expansion problem. The integral form for the coefficients

$$
A_{n}=\frac{2}{\pi\left(1+\delta_{0}^{n}\right)} \int_{0}^{\pi}\left(s_{0}-\cos \theta\right)^{1 / 2} \cos n \theta d \theta,
$$

(where $\delta_{0}^{n}=1$ for $n=0$, and is zero otherwise) may be evaluated using an integral representation for the
Legendre functions. The result is

$$
A_{n}=\frac{\sqrt{2}}{\pi\left(1+\delta_{0}^{n}\right)} \frac{\left(s_{0}^{2}-1\right)^{1 / 2}}{\left(n^{2}-\frac{1}{4}\right)} Q_{n-1 / 2}^{1}\left(s_{0}\right) .
$$

Equations (11) and (12) thus specify the magnetic field in terms of the induced current $I_{m}$.

The electric field may be determined from the magnetic field using the relation

$$
\sigma \vec{E}=\nabla \times \vec{H}
$$

in which $\sigma$ is the conductivity of the highly conducting medium. Using the Legendre function recurrence formulas, one may express the $\theta$ component as

$$
\begin{gathered}
E_{\theta}=-\sum_{n=0}^{\infty}\left[\frac { b _ { n } \operatorname { c o s } n \theta } { \sigma a } \left\{(s-\cos \theta)^{3 / 2}\left(n^{2}-\frac{1}{4}\right) P_{n-1 / 2}(s)\right.\right. \\
\left.\left.-\frac{1}{2}(s-\cos \theta)^{1 / 2}\left(s^{2}-1\right) P_{n-1 / 2}^{1}(s)\right\}\right],
\end{gathered}
$$

with

$$
b_{n}=\frac{\sqrt{2} I_{m}}{2 \pi^{2} a\left(1+\delta_{0}^{n}\right)\left(n^{2}-\frac{1}{4}\right)} \cdot \frac{Q_{n-1 / 2}^{1}\left(s_{0}\right)}{P_{n-1 / 2}^{1}\left(s_{0}\right)} .
$$

The voltage per turn is given by

$$
\frac{V}{N}=+\oint \vec{E} \cdot \overrightarrow{d l}=+\int_{-\pi}^{\pi}\left[E_{\theta} h_{\theta}\right]_{s=s_{0}} d \theta,
$$

where the metric coefficient

$$
h_{\theta}=\frac{a}{s-\cos \theta} .
$$

Performing this integration and using the properties of the Legendre functions, one finds that

$$
R_{m}=\frac{-(V / N)}{I_{m}}=\frac{S_{A}\left(s_{0}\right)}{\sigma \rho_{0}},
$$

where

$$
S_{A}\left(s_{0}\right)=\frac{s_{0}}{\left(s_{0}^{2}-1\right)^{1 / 2}} \sum_{n=0}^{\infty} \frac{-1}{\pi^{2}\left(1+\delta_{0}^{n}\right)\left(n^{2}-\frac{1}{4}\right)} \frac{Q_{n-1 / 2}^{1}\left(s_{0}\right)}{P_{n-1 / 2}^{1}\left(s_{0}\right)} .
$$

Note that as

$$
s_{0}=\frac{\rho_{0}}{r_{0}}
$$

the factor $S_{A}$ depends only upon the toroid dimensions. The variation of $S_{A}$ with $r_{0} / \rho_{0}$ is illustrated in figure 3 . 


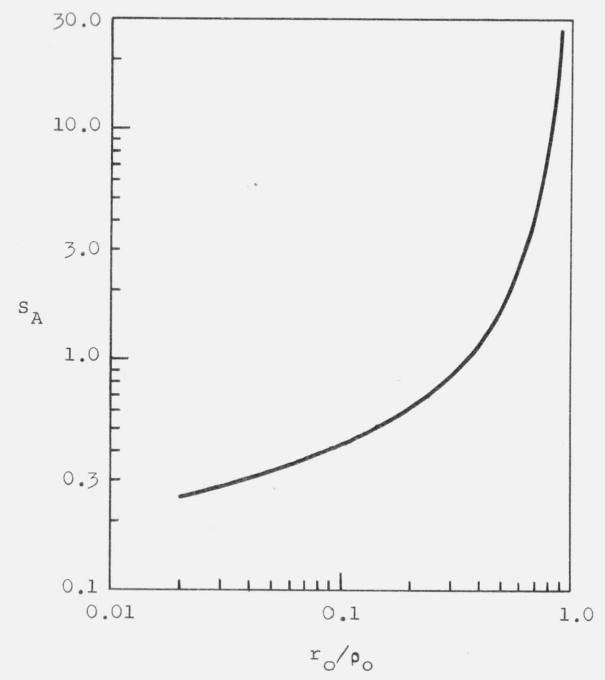

FIGURE 3. Variation of $\mathrm{S}_{\mathrm{A}}$ with toroid dimension ratio.

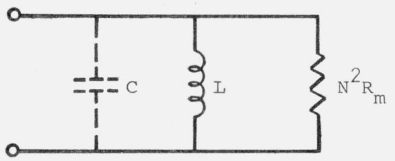

FIGURE 4. Equivalent circuit for the admittance of the toroid antenna.

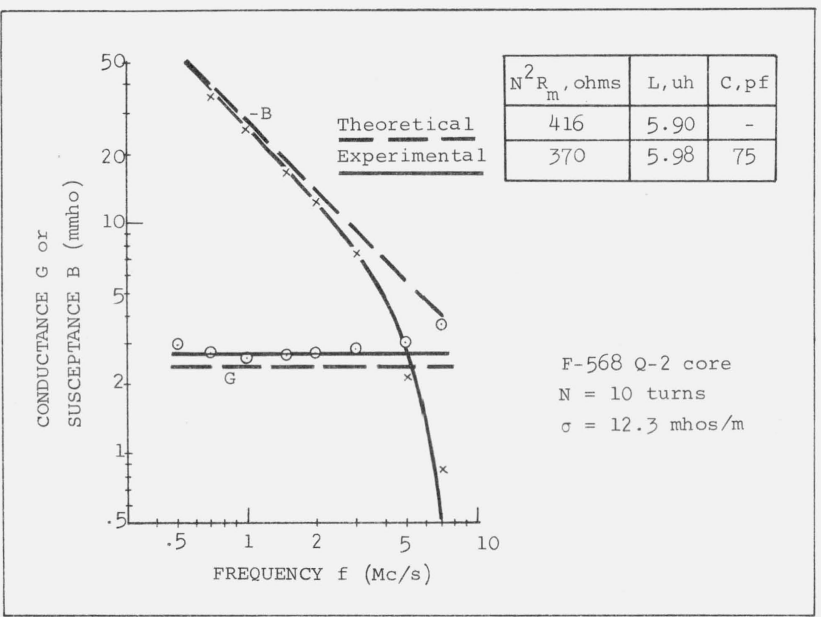

Figure 5. Comparison of the measured and theoretical conductance and susceptance of a toroid antenna model.
Using (14), one may eliminate $I_{m}$ from (3) and solve for the ratio of $I$ to $V$ to obtain the driving-point admittance

$$
Y=\frac{1}{N^{2} R_{m}}+\frac{1}{j \omega L}
$$

where

$$
L=\frac{\mu_{c} A_{c} N^{2}}{2 \pi \rho} .
$$

The value of $L$ is seen to be the inductance one would expect a toroid of $N$ turns in a lossless medium to have. Besides the resistive and inductive terms in (16), the driving-point admittance may include a capacitive term, $j \omega C$, arising from the turn-to-turn and turn-to-medium stray capacity inherent in the driving winding, or from a capacitance deliberately added at the driving point for the purpose of tuning the antenna. An equivalent circuit for the toroid antenna is thus as shown in figure 4.

The circuit of figure 4 may be regarded as a simplified equivalent circuit for a transformer. Indeed, one may think of the toroid as a transformer with the $N$ driving winding turns forming a primary, and the conducting medium linking the toroid forming a combined one-turn secondary and resistive load. The process of relating $I_{m}$ to $V / N$ may thus be considered as a method for determining the resistive load $R_{m}$ in the transformer secondary circuit.

The admittances of several model toroid antennas in a conducting medium were measured as a check on the theoretical admittance as given by (16). Conducting media were modeled by aqueous solutions of sodium chloride with conductivities in the range 2.8 to 12.3 mhos per meter. Frequencies in the range 0.5 to $7.0 \mathrm{Mc} / \mathrm{s}$ were used. This range was low enough that the antenna models were electrically small in the conducting medium, but high enough that any signals reflected from the sides or bottom of the solution tank were greatly attenuated. In regard to the assumption that as far as the admittance was concerned, one could ignore the proximity of the surface, it was found that as long as the model was completely submerged, the observed change in admittance with depth was less than one percent. Figure 5 shows a sample of the measured and theoretical conductances and susceptances for one of the toroids. It can be seen that the experimental values can be fitted well by the admittance of a parallel $R-L-C$ circuit. The values of $R$ and $L$, so obtained, agree closely with the values obtained from (16); the presence of $C$ indicates that the effects of stray capacity were beginning to be important at the frequencies used.

\section{Radiating Properties of the Toroid Antenna}

Given the current moment of a small antenna, one can determine its effective length; once the effective 
length is known, it is a straightforward matter to compute other antenna merit factors, such as effective area and gain. The first objective of this section is then to determine the current moment produced by the toroid antenna.

As far as the fields outside the toroid antenna are concerned, the source may be replaced by a set of equivalent source currents flowing in a surface lying immediately outside of the volume formerly occupied by the toroid. In order that the external fields remain unchanged, one selects electric surface currents $\overrightarrow{J_{s}}$ and magnetic surface currents $\vec{K}_{s}$ such that

$$
\begin{aligned}
& \overrightarrow{J_{s}}=\vec{n} \times \vec{H} \\
& \vec{K}_{s}=\vec{E} \times \vec{n},
\end{aligned}
$$

where $\vec{n}$ is the unit outward normal to the surface. As the effective electric surface currents circulate around only the toroid cross section, whereas the effective magnetic surface currents circulate along the toroid, one suspects that the contribution to the distant fields from the electric currents is small compared with that from the magnetic currents. It can be shown that the ratio of contributions of electric to magnetic equivalent sources is of the order of $\pi r_{0}^{2} / \delta^{2}$ for the range of dimensions $0.04<r_{0} / \rho_{0}<0.8$, where $\delta$ is the skin depth in the conducting medium and the dimensions $\rho_{0}$ and $r_{0}$ are illustrated in figure 1 . Thus for the small toroid antenna, the contributions from the electric equivalent source currents may be neglected. The total magnetic effective source current circulating around the toroid, one observes from (18) is,

$$
\oint_{C} K_{s} d s=\frac{V}{N}
$$

where $C$ is a contour on the surface lying perpendicular to the direction of magnetic surface current flow.

Whereas the magnetic current moment $(V l)$ produced by a small loop of radius $\rho$ carrying an electric current $I_{e}$ is given by

$$
(V l)=j \omega \mu\left(\pi \rho^{2}\right) I_{e},
$$

and it follows by duality that the electric current moment of a small loop of radius $\rho$ carrying a magnetic current $V_{m}$ is given by

$$
(I l)=-j \omega \underline{\underline{\epsilon}}\left(\pi \rho^{2}\right) V_{m},
$$

where $\epsilon=\epsilon+\sigma /(j \omega)$.

For the magnetic toroid antenna, one may estimate the current moment as

$$
(I l)=-\sigma\left(\pi \rho_{e}^{2}\right) \frac{V}{N},
$$

since for a highly conducting medium, $j \omega \epsilon \approx \sigma$, and one may consider the distributed magnetic current $K_{s}$ as being replaced by the total magnetic current from (19), concentrated at an effective radius $\rho_{e}$.

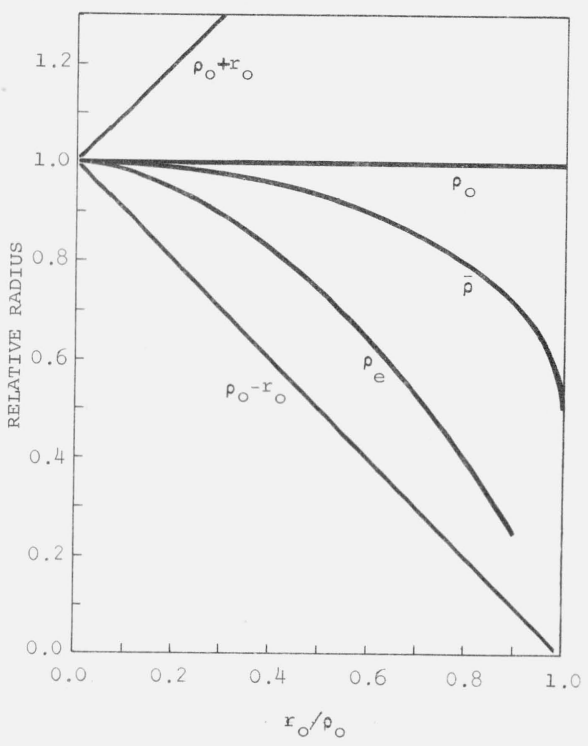

FiguRE 6. Variation of the effective radius $\left(\rho_{\mathrm{e}}\right)$ and the effective magnetic radius $(\bar{\rho})$ with toroid dimension ratio.

Using the tangential electric field found previously in (13), one can determine the effective radius from

$$
\begin{aligned}
\rho_{e}^{2} & =\frac{\oint \rho^{2} K_{s} d s}{\oint K_{s} d s} \\
& =\frac{\int_{-\pi}^{\pi}\left[\frac{a^{2}\left(s^{2}-1\right)}{(s-\cos \theta)^{2}} E_{\theta} h_{\theta}\right]_{s=s_{0}} d \theta}{\int_{-\pi}^{\pi}\left[E_{\theta} h_{\theta}\right]_{s=s_{0}} d \theta} \\
& =\rho_{0}^{2} \frac{S_{B}\left(s_{0}\right)}{S_{A}\left(s_{0}\right)},
\end{aligned}
$$

where

$$
\begin{aligned}
S_{B}\left(s_{0}\right)=-\frac{4\left(s_{0}^{2}-1\right)}{\pi^{2} s_{0}} \sum_{n=0}^{\infty} \frac{Q_{n-1 / 2}^{1}\left(s_{0}\right)}{\left(1+\delta_{0}^{n}\right)} & {\left[\frac{Q_{n-1 / 2}^{1}\left(s_{0}\right) P_{n-1 / 2}\left(s_{0}\right)}{P_{n-1 / 2}^{1}\left(s_{o}\right)}+\frac{Q_{n-1 / 2}^{2}\left(s_{0}\right)}{3\left(n^{2}-\frac{1}{4}\right)}\right], }
\end{aligned}
$$

and $\mathrm{S}_{A}\left(\mathrm{~s}_{0}\right)$ is given by (15). The variation of $\rho_{e} / \rho_{0}$ with the dimension ratio $r_{0} / \rho_{0}=1 / s_{0}$ is shown in figure 6 .

One may put the antenna moment of (21) in terms of its input current by using the admittance given by (16). Then since the effective length is the ratio of the current moment to the input current, one finds that the effective length for the toroid antenna is

$$
l_{e}=\frac{-\pi \sigma \rho_{e}^{2}}{N Y} \text {. }
$$

An interesting special case occurs when the shunt capacity (stray capacity plus external capacitance at the antenna terminals) is such as to resonate the 
antenna. Then

$$
Y=\frac{1}{N^{2} R_{m}}
$$

and

$$
l_{e}=-\pi N \rho_{0} S_{B}\left(s_{0}\right) \cdot
$$

The magnitude of the effective length in (25) is the maximum magnitude that can be obtained by varying the external capacity. The minus sign in (25) (or the angle associated with the complex quantity in (24)) merely indicates that there is a difference in phase between the incident wave and the open-circuit voltage appearing on the antenna terminals. As $S_{B}$ depends only upon the ratio $s_{0}=\rho_{0} / r_{0}$, one sees that the effective length for this special case is independent of the conductivity or other characteristics of the medium. The variation of the magnitude of the effective length for the resonant antenna with change in the dimension ratio $r_{0} / \rho_{0}$ is shown in figure 7 .

The resonant toroid antenna appears suitable for use as a probe for measuring electric fields in a highly conducting medium. Its dual, the insulated loop antenna, may be used to measure magnetic fields in such a medium. These antennas share the property that the open circuit voltage available for a given signal may be increased without increasing the size of the antenna by increasing the number of turns.

When the antenna is used as a transmitter, the equivalent admittance presented to the source is essentially $Y$, the driving-point admittance of the antenna. On the other hand, when the antenna is used as a receiver, the equivalent source (of the Norton type) presented to the load consists of the admittance $Y$ in parallel with a current generator of current $I_{s c}$. In terms of the open circuit voltage $V_{\text {oc }}$, or the effective length $l_{e}$ and the incident field strength $E_{i}$,

$$
I_{s c}=Y V_{o c}=Y l_{e} E_{i} .
$$

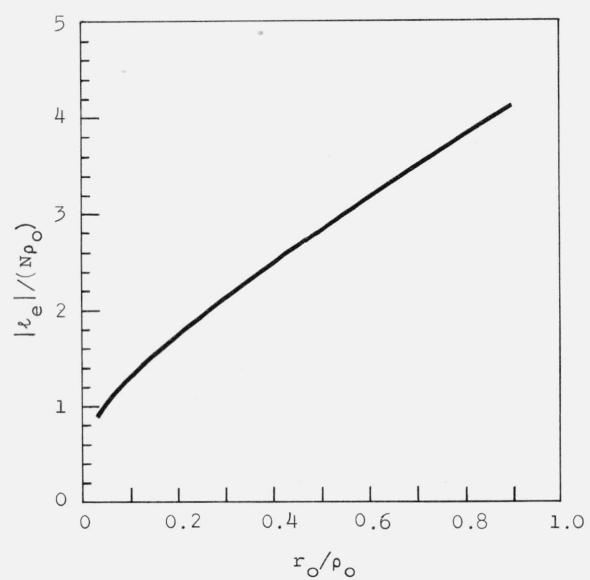

FIGURE 7. Normalized magnitude of the effective length for the resonant toroid antenna.
For the toriod antenna, it follows from (24) and (22) that

$$
I_{s c}=\frac{-\pi \sigma \rho_{e}^{2}}{N} E_{i}=\frac{-\pi \sigma \rho_{0}^{2}}{N}\left(S_{B} / S_{A}\right) E_{i} .
$$

One may then determine from (26), (16), and (14) that the available power at the antenna terminals is

$$
P_{a}=\frac{\left|I_{s c}\right|^{2}}{8 G}=\frac{1}{8} \pi^{2} \sigma^{2} \rho_{e}^{4} R_{m}\left|E_{i}\right|^{2}=\frac{1}{8} \pi^{2} \sigma \rho_{0}^{3}\left(S_{B}^{2} / S_{A}\right)\left|E_{i}\right|^{2},
$$

where $G$ is the real part of the antenna admittance, $Y$. The power density of the incident wave in a highly conducting medium is approximately given by

$$
W_{i}=\frac{\left|E_{i}\right|^{2}}{2 \omega \mu \delta}=\frac{1}{4} \sigma \delta\left|E_{i}\right|^{2} .
$$

It follows that the effective area of the toroid antenna is

$$
A_{e}=\frac{P_{a}}{W_{i}}=\frac{\pi^{2} \rho_{e}^{4} R_{m}}{\omega \mu \delta^{3}}=\frac{1}{2} \pi^{2} \delta^{-1} \rho_{0}^{3}\left(S_{B}^{2} / S_{A}\right) .
$$

If the antenna is just beneath the surface of the highly conducting medium, it may be advantageous to compare the power available at the antenna to the power density $W_{s}$ of the incident wave above the surface. It can be seen that the relative effective area

$$
A_{r}=\frac{P_{a}}{W_{s}}
$$

(designated just "effective area" by Hansen [1963]) is related to the effective area $A_{e}$ by the ratio of power densities $W_{i} / W_{s}$. For a plane surface wave (such as the one discussed by Jordan [1950]) refracting into a highly conducting medium,

$$
W_{i} / W_{s}=\pi \delta / \lambda,
$$

where $\lambda$ is the wavelength in the dielectric half space. Consequently, the relative effective area of the toriod antenna is

$$
A_{r}=\frac{1}{2} \pi^{3} \sigma \lambda^{-1} \rho_{e}^{4} R_{m}=\frac{1}{2}\left(\pi \rho_{0}\right)^{3} \lambda^{-1}\left(S_{B}^{2} / S_{A}\right) .
$$

This quantity may be compared directly with the effective area for an antenna above the surface.

The frequency dependence of the relative effective area of the toroid antenna is the same as that of the relative effective area of an electric-dipole-in-radome antenna discussed by Hansen [1963]. Further comparison indicates that the relative effective areas of the two antenna types are of the same order of magnitude for antennas with the same maximum exterior dimension. It follows that the relative effective area of a thin toroid antenna, $r_{0} \ll \rho_{0}$, may be much greater than the relative effective area of an electric-dipolein-radome antenna whose radome occupies the same volume. 


\section{Conclusions}

The driving-point admittance of the small toroid antenna is found to vary with frequency like a parallel $R-L-C$ circuit. If the toroid is conceived as a transformer with the conducting medium forming a combined one-turn secondary winding and resistive load, $R$ is the load impedance as reflected into the primary circuit, $L$ is the inductance of the primary winding, and $C$ represents the low-frequency effects of the turn-toturn and turn-to-medium stray capacity. In the same manner that a small loop of electric current may be considered as a magnetic current dipole for determining distant fields, the small toroid antenna may be considered as an electric current dipole. The moment is directed along the axis of rotational symmetry of the toroid. The effective length of the tuned toroid antenna is found to be independent of the characteristics of the conducting medium, and hence the toroid may be used as a probe for measuring the electric field in such a medium. The relative effective area of an antenna just below the surface in a highly conducting medium is the ratio of the available power at the antenna terminals to the power density in an incident wave above the surface. The relative effective area of the toroid antenna is found to be similar in frequency dependence and of the same order of magnitude as the relative effective area of a small electric dipole in a radome, if the outside dimensions of the antennas are equal.
This work was supported by the Office of Naval Research under Contract Nonr 2798(01)(FBM). The author also wishes to gratefully acknowledge the suggestions and encouragement of R. H. Williams, of the University of New Mexico.

\section{References}

Erdelyi, A., W. Magnus, F. Oberhettinger, and F. G. Tricomi (1953), Higher Transcendental Functions, 1, 123, 156, 160-161, 173 (McGraw-Hill Book Co., Inc., New York, N.Y.).

Hansen, R. C. (May 1963), Radiation and reception with buried and submerged antennas, IEEE Trans. Ant. Prop. AP-11, No. 3, 207-216.

Jordan, E. C. (1950), Electromagnetic Waves and Radiating Systems, 201-209 (Prentice-Hall, Inc., Englewood Cliffs, N.J.).

Moore, R. K., and W. E. Blair (Nov.-Dec. 1961), Dipole radiation in a conducting half-space, J. Res. NBS 65D (Radio Prop.), No. 6, 547-563.

Wait, J. R. (Nov. 1961), The electromagnetic fields of a horizontal dipole in the presence of a conducting half-space, Can. J. Phys. 39, No. 11, 1017-1028.

(Paper 69D4-501) 